TRANSACTIONS OF THE

AMERICAN MATHEMATICAL SOCIETY

Volume 319, Number 1, May 1990

\title{
ALMOST SPLIT SEQUENCES AND ZARISKI DIFFERENTIALS
}

\author{
ALEX MARTSINKOVSKY
}

\begin{abstract}
Let $R$ be a complete two-dimensional integrally closed analytic $k$ algebra. Associated with $R$ is the Auslander module $A$ from the fundamental sequence $0 \rightarrow \omega_{R} \rightarrow A \rightarrow R \rightarrow k \rightarrow 0$ and the module of Zariski differentials $D_{k}(R)^{* *}$. We conjecture that these modules are isomorphic if and only if $R$ is graded. We prove this conjecture for (a) hypersurfaces $f=X_{3}^{n}+g\left(X_{1}, X_{2}\right)$, (b) quotient singularities, and (c) $R$ graded Gorenstein.
\end{abstract}

\section{INTRODUCTION}

Let $(R, \mathfrak{m})$ be a complete two-dimensional integrally closed nonregular local domain with dualizing module $\omega_{R}$ and let $\mathfrak{R e f}(R)$ denote the category of reflexive $R$-modules. In [Aus1] Auslander proves that $\mathfrak{R e f}(R)$ has almost split sequences and that they can be constructed by means of a simple procedure.

More precisely, since $\operatorname{Ext}_{R}^{2}\left(R / \mathfrak{m}, \omega_{R}\right) \cong R / \mathfrak{m}$, all exact sequences

$$
0 \rightarrow \omega_{r} \stackrel{q}{\longrightarrow} A \stackrel{p}{\longrightarrow} R \rightarrow R / \mathfrak{m} \rightarrow 0
$$

representing nonzero elements are isomorphic. It is not difficult to show that the module $A$ is reflexive [Aus2, Theorem 3.6, p. 227] and that (0.1) has properties analogous to those of an almost split sequence. Namely, for any reflexive module $X$ any map $g: X \rightarrow R$ which is not a splittable epimorphism can be lifted to $A$ via $p$ [Aus1, p. 527]. Dually, for any reflexive module $Y$ any map $g: \omega_{R} \rightarrow Y$ which is not a splittable monomorphism can be extended to $A$ via $q$. If $C$ and $B$ are reflexive $R$-modules let $C \cdot B$ denote the tensor product of $C$ and $B$ in $\mathfrak{R e f}(R)$, i.e., $C \cdot B:=\left(C \otimes_{R} B\right)^{* *}$, where "*" stands for $\operatorname{Hom}_{R}(-, R)$. The aforementioned result of Auslander can now be stated as follows (see [Aus1, Theorem 6.6; Aus3, Proposition 1.1; AR, Theorem 3.6 of Part 1]):

Received by the editors August 9, 1988.

1980 Mathematics Subject Classification (1985 Revision). Primary 14J17; Secondary 16A03.

Key words and phrases. Zariski differentials, Auslander module, almost split sequences, fundamental sequence, moduli algebra, matrix factorizations, graded analytic $k$-algebras, reflexive modules, isolated hypersurface singularities.

This paper is part of the author's Ph.D. thesis submitted at Brandeis University. 
Theorem. Let $D$ be an indecomposable reflexive $R$-module which is not projective. Then

(a) $0 \rightarrow D \cdot \omega_{R} \stackrel{\text { id } \cdot p}{\longrightarrow} D \cdot A \stackrel{\text { id } \cdot p}{\longrightarrow} D \cdot R=D \rightarrow 0$ is exact.

(b) The exact sequence $0 \rightarrow D \cdot \omega_{R} \rightarrow D \cdot A \rightarrow D \rightarrow 0$ is either split or almost split.

(c) $0 \rightarrow D \cdot \omega_{R} \rightarrow D \cdot A \rightarrow D \rightarrow 0$ is almost split if rank $D$ is not divisible by the characteristic of $R / \mathfrak{m}$.

(d) If $R / \mathfrak{m}$ is algebraically closed then $0 \rightarrow D \cdot \omega_{R} \rightarrow D \cdot A \rightarrow D \rightarrow 0$ is almost split if and only if $\operatorname{rank} D$ is not divisible by the characteristic of $R / \mathfrak{m}$.

Thus if $R / \mathfrak{m}$ is of characteristic 0 all almost split sequences in $\mathfrak{R e f}(R)$ can be obtained from $(0.1)$ by taking the reflexive tensor product. It is for this reason that $(0.1)$ is called the fundamental exact sequence and the study of this sequence becomes critically important for a better understanding of the category $\mathfrak{R e f}(R)$. Thus we are led to the study of the module $A$ which, following a suggestion of $\mathrm{K}$. Behnke, we call the Auslander module of $R$. In particular it becomes desirable to identify this module in some sense and to be able to determine whether this module is a new entity or something already known but camouflaged by abstract module-theoretic properties. And these properties are truly amazing. We mention one of them, present when $R$ is Gorenstein, i.e., $\omega_{R}$ is isomorphic to $R$. It is then an easy consequence of the map-extending property of the fundamental sequence that $A$ covers any reflexive module $X$ which has no free summands, i.e., a direct sum of copies of $A$ can be mapped onto $X$. Notice that $A$ itself has no free summands. Indeed if $A \cong M \amalg R$ then (0.1) shows that $\operatorname{rank} M=1$ and the divisor of $M$ (i.e., the corresponding element in the divisor class group of $R$ ) is trivial. Since $A$ is reflexive we would have $M \cong R$ and (0.1) would give us a finite projective resolution of the residue field, contrary to the nonregularity of $R$. Let us now decompose $A$ into a direct sum of indecomposables $A \cong \bigsqcup_{i \in I} A_{i}$ (since $\left.\operatorname{rank} A=2, \operatorname{card}(I) \leq 2\right)$. Let $\left\{A_{j} \mid j \in J\right\}$ be the complete set of nonisomorphic direct summands of $A$. Then the modules $A_{j}, j \in J$, cover any reflexive module without free summands and this property characterizes the module $A$. We should remark that if $R$ is Gorenstein and not isomorphic to the singularity of type $A_{n}$ then $A$ is indecomposable.

We restrict our attention to those $R$ which are analytic $k$-algebras, i.e., quotients of formal power series rings over a field $k$.

In $\S 1$ we begin the study of the Auslander module when $R$ is a (complete) two-dimensional quotient singularity, i.e., $R$ is the invariant subring $S^{G}$ of the formal power series ring $S:=k\left[\left|X_{1}, X_{2}\right|\right]$ over a field $k$ and $G$ a finite group of $k$-algebra automorphisms of $S$. We assume, in addition, that the order of $G$ is invertible in $k$ and that $R$ is unramified in $S$ at the height 1 primes (this means that for any height 1 prime $p$ of $R$ the extension $R_{p} \rightarrow S_{p}$ is unramified). We then show that in this case the Auslander module is isomorphic to the bidual 
$D_{k}(R)^{* *}$ of the (finitely generated) module of Kähler differentials of $R$ over $k$. Although this result has been known to specialists for some time it is not easy to find it in the literature and for the convenience of the reader we provide a proof (of a slightly more general result).

The above observation leads to the following questions. Suppose $R$ is a complete two-dimensional integrally closed analytic $k$-algebra over a field $k$.

Question 1. Is it always true that the Auslander module is isomorphic to the module $D_{k}(R)^{* *}$ of Zariski differentials?

Question 2. If the answer to Question 1 is "no" when are these modules isomorphic?

The presence of an isomorphism between $A$ and $D_{k}(R)^{* *}$ is important for two reasons.

First is that $A$ acquires properties of $D_{k}(R)^{* *}$, which leads to the following "differential" description of the middle term of the almost split sequence ending with an indecomposable reflexive $F$. Let $D(-)$ denote the duality $\operatorname{Hom}_{R}\left(-, \omega_{R}\right): \operatorname{Ref}(R) \rightarrow \operatorname{Ref}(R)$. From $(0.1)$ we have $A \cong D(A)$. Computing now the middle term of the almost split sequence we have

$$
\begin{aligned}
F \cdot A & =(F \otimes A)^{* *} \cong \operatorname{Hom}_{R}\left(F^{*}, A\right) \cong \operatorname{Hom}_{R}\left(F^{*}, \operatorname{Hom}_{R}\left(A, \omega_{R}\right)\right) \\
& \cong \operatorname{Hom}_{R}\left(F^{*} \otimes A, \omega_{R}\right) \cong \operatorname{Hom}_{R}\left(A, \operatorname{Hom}_{R}\left(F^{*}, \omega_{R}\right)\right) \\
& \cong \operatorname{Hom}_{R}\left(D_{k}(R)^{* *}, D\left(F^{*}\right)\right) \cong \operatorname{Hom}_{R}\left(D_{k}(R), D\left(F^{*}\right)\right) \cong \operatorname{Der}_{k}\left(R, D\left(F^{*}\right)\right) \\
& =\text { the } R \text {-module of } k \text {-derivations of } R \text { into } D\left(F^{*}\right) .
\end{aligned}
$$

The other reason is that $D_{k}(R)^{* *}$ acquires module-theoretic properties of $A$ and can be characterized by these properties. In particular, if, in addition, $R$ is Gorenstein then $D_{k}(R)^{* *}$ is characterized by the property that it covers reflexive modules without free summands.

In $\S 2$ we give a general criterion: $A$ is isomorphic to $D_{k}(R)^{* *}$ if and only if $D_{k}(R)^{* *}$ can be mapped onto the maximal ideal $\mathfrak{m}$ of $R$. This implies that if $R$ is graded Gorenstein then $A \cong D_{k}(R)^{* *}$. Notice however that it is not necessary for $R$ to be Gorenstein in order for $A$ and $D_{k}(R)^{* *}$ be isomorphic. Indeed if $R$ is a quotient singularity then $A \cong D_{k}(R)^{* *}$. But if $G$ is not a subgroup of $\operatorname{SL}(2, k)$ then $R$ is not Gorenstein. This leads us to the following conjecture:

Conjecture. Suppose $R$ is a two-dimensional integrally closed analytic $k$-algebra over an algebraically closed field $k$ of characteristic 0 . Then $A \cong D_{k}(R)^{* *}$ if and only if $R$ is graded.

The main result of this paper is a proof of this conjecture for the isolated hypersurface singularities $k\left[\left|X_{1}, X_{2}, X_{3}\right|\right] /(f)$, where $f$ is of the form $X_{3}^{n}+$ $g\left(X_{1}, X_{2}\right)$ and $g \in k\left[\left|X_{1}, X_{2}\right|\right]$. In particular we obtain a negative answer to Question 1. The statement of the main theorem can be found in $\S 3$, where we also mention further evidence in support of the conjecture. 
The proof of the main theorem is postponed until $\S 8$. In the preceding sections we prove auxiliary results enabling us to produce presentation matrices of the modules $A$ and $D_{k}(R)^{* *}$.

In $\S 4$ we prove an "acyclicity lemma" which helps to construct projective resolutions over integrally closed two-dimensional hypersurface rings. With its aid we obtain a presentation matrix for the module $A$.

In $\S 5$ we show that the module $D_{k}(R)^{* *}$ of Zariski differentials of a twodimensional isolated hypersurface singularity $R:=S /(f)$ is isomorphic to the module of $k$-derivations $D_{k}(R)^{*}$ of $R$ into itself. Therefore, $D_{k}(R)^{* *}$ is isomorphic to the second syzygy module of the moduli algebra $R / \overline{j(f)}$ of $f$, where $\overline{j(f)}$ is the image of the jacobian ideal $j(f)$ of $f$ in $R$. Thus to obtain a presentation matrix of the module $D_{k}(R)^{* *}$ we need to construct a projective resolution of the moduli algebra $R / \overline{j(f)}$. This remains an unsolved problem for a general $f$. We take on the case $f:=X_{3}^{n}+g\left(X_{1}, X_{2}\right)$.

In $\S 6$ we solve this problem in dimension 1, i.e., for an isolated singularity of a formal plane curve. Information which we obtain enables us, in $\S 7$, to produce a minimal projective resolution of $R / \overline{j(f)}$, where $f$ is of the form $X_{3}^{n}+$ $g\left(X_{1}, X_{2}\right)$. An important part of our approach is a construction of Eisenbud, called matrix factorization of $f$, which describes the periodic parts of projective resolutions over hypersurface rings.

Finally in $\S 8$ we give a proof of the main theorem by comparing the presentation matrices of $E$ and $D_{k}(R)^{* *}$. Here we use a crucial result of Scheja and Wiebe which asserts that the existence of a nonnilpotent derivation of $R$ into itself implies that $R$ is graded.

The author expresses his deep gratitude to M. Auslander for posing the original problem and stimulating encouragement during the work on this paper. The author also thanks R.-O. Buchweitz and J. Wahl for valuable comments.

\section{QUOTIENT SINGULARITIES}

For the rest of this paper, by an analytic $k$-algebra we will understand a quotient of a formal power series ring over field $k$. For an analytic $k$-algebra $R$, the module of Kähler differentials of $R$ over $k$ will be denoted by $D_{k}(R)$. This is a finitely generated $R$-module together with a $k$-derivation $d_{R / k}: R \rightarrow D_{k}(R)$ (whose image generates $D_{k}(R)$ ) having the universal factorization property with respect to the $k$-derivation of $R$ into finitely generated $R$-modules. Such a module exists for any analytic $k$-algebra [Sch]. Our goal in this section is to show that the Auslander module $A$ from the fundamental exact sequence $(0.1)$ of a complete 2-dimensional quotient singularity $R$ is isomorphic to the module of Zariski differentials of $R$ (i.e., the bidual $D_{k}(R)^{* *}$ of the module of Kähler differentials of $R$ ).

First we need a few general facts about finite extensions of analytic $k$ algebras. 
Lemma 1.1. Let $R \rightarrow S$ be a module-finite extension of integrally closed analytic $k$-algebras unramified at the height 1 primes of $R$. Consider the homomorphism of $S$-modules

$$
v: D_{k}(R) \otimes_{R} S \rightarrow D_{k}(S)
$$

given by $v\left(d_{R / k}(r) \otimes s\right):=s d_{S / k}(r)$, where $r \in R$ and $s \in S$ with $d_{R / k}$ and $d_{S / k}$ being the universally finite derivations. Then the homomorphism

$$
v^{*}: \operatorname{Hom}_{S}\left(D_{k}(S), S\right) \rightarrow \operatorname{Hom}_{S}\left(D_{k}(R) \otimes_{R} S, S\right)
$$

is an isomorphism.

Proof. The homomorphism $v^{*}$ is a map between reflexive $S$-modules. A module $M$ over an integrally closed domain of dimension $\geq 2$ is reflexive if and only if $M$ is torsion-free and depth $M \geq 2$. Therefore, $v^{*}$ is a map between reflexive $R$-modules. Thus it suffices to show that the localization $v_{p}^{*}$ of $v^{*}$ at any height 1 prime $p$ of $R$ is an isomorphism. To this end consider the exact sequence

$$
0 \rightarrow \operatorname{ker} v \rightarrow D_{k}(R) \otimes_{R} S \stackrel{v}{\longrightarrow} D_{k}(S) \rightarrow D_{R}(S) \rightarrow 0
$$

(the term $D_{R}(S)$ makes sense because $S$ is finite over $R$ ). Since $D_{R}(S)$ is generated by the image of the universal derivation $d_{S / R}$, the localization $D_{R}(S)_{P}$ is generated by the image of $\left(d_{S / R}\right)_{p}$. Since $\left(d_{S / R}\right)_{p}$ is an $R_{p}$-derivation into a finitely generated $S_{p}$-module and since $R_{p}$ is unramified in $S_{p}$ we have $\left(d_{S / R}\right)_{p}=0\left[\mathrm{AB}\right.$, Theorem 2.5] and, therefore, $D_{R}(S)_{p}=0$. On the other hand, $\operatorname{rank}_{R} D_{k}(R)=\operatorname{rank}_{S} D_{k}(S)[\mathrm{P} 1,1.2]$, therefore, $\operatorname{ker} v$ is a torsion $S$ module and $\left(v^{*}\right)_{p}$ is an isomorphism. The lemma is proved.

Lemma 1.2. Let $S$ be an integrally closed analytic $k$-algebra, $G$ a finite group of $k$-algebra automorphisms of $S$ and $R:=S^{G}$ the ring of invariants. Then

(a) $S$ is a finite $R$-module;

(b) $R$ is an integrally closed analytic k-algebra;

(c) $D_{k}(S)$ is a module over the twisted group ring $S[G]$.

Proof. It is well known [B-AC, Chapter V, $\S 1$, No. 9, Proposition 22] that $S$ is integral over $R$ and that $R$ is integrally closed. Let $L$ (resp., $K$ ) be the field of quotients of $S$ (resp., $R$ ). Then by [ibid, Proposition 23, Corollary] $K=L^{G}$. Hence $L$ is a finite-dimensional separable field extension of $K$. In Nagata's terminology [Nag, Chapter 1, No. 10], $S$ is an almost finite separable integral extension of the noetherian normal ring $R$. By [ibid, Corollary 10.16] $S$ is a finite $R$-module. This proves (a). Since $S$ is a finite $R$-module, $R$ is a complete local ring [B-AC, Chapter III, $\S 3$, Example 18b]. From the theory of complete local rings we know that $R$ is a quotient of a formal power series ring over $k$ [B-AC', Chapter IX, $\S 3$, No. 3, Theorem 2]. This proves (b). Finally, the universal property of $D_{k}(S)$ implies that each $g \in G$ induces a map $g: D_{k}(S) \rightarrow D_{k}(S)$ such that $g(s m)=g(s) g(m)$ for each $s \in S$ and $m \in D_{k}(S)$. This means that $D_{k}(S)$ is an $S[G]$-module. The lemma is proved. 
Proposition 1.3. Let $S$ be an integrally closed analytic k-algebra, $G$ a finite group of $k$-algebra automorphisms and $R:=S^{G}$ the ring of invariants. Assume furthermore that the order of $G$ is invertible in $k, R$ is unramified in $S$ at the height 1 primes and the $S$-module $D_{k}(S)$ is reflexive. Then the $R$-modules $D_{k}(S)^{G}$ and $D_{k}(R)^{* *}$ are isomorphic (here “*” means $\operatorname{Hom}_{R}(-, R)$ ).

Proof. Applying Lemma 1.1 we obtain an isomorphism of $S$-modules

$$
v^{*}: \operatorname{Hom}_{S}\left(D_{k}(S), S\right) \stackrel{\sim}{\rightarrow} \operatorname{Hom}_{S}\left(D_{k}(R) \otimes_{R} S, S\right) .
$$

If we define an action of $G$ on $D_{k}(R) \otimes_{R} S$ by letting $G$ act on $S$ only and consider the usual action of $G$ on the Hom-sets given by $f \rightarrow g \circ f \circ$ $g^{-1}$, where $f \in \operatorname{Hom}_{S}(-,-)$ and $g \in G$, then $v^{*}$ becomes an isomorphism of $S[G]$-modules. Since the order of $G$ is invertible in $k$ the fixed point functor is exact. Therefore, $\left(v^{*}\right)^{G}$ is an isomorphism between the $R$-modules $\operatorname{Hom}_{S}\left(D_{k}(S), S\right)^{G}$ and $\operatorname{Hom}_{S}\left(D_{k}(R) \otimes_{R} S, S\right)^{G}$. We now claim that the fixed point functor commutes with the above Hom's. Indeed if $M$ and $N$ are finitely generated $S[G]$-modules and $N$ is reflexive as an $S$-module then the restriction map

$$
\begin{array}{r}
\operatorname{Hom}_{S[G]}(M, N)=\operatorname{Hom}_{S}(M, N)^{G} \rightarrow \operatorname{Hom}_{R}\left(M^{G}, N^{G}\right): f \mapsto f \mid M^{G}, \\
f \in \operatorname{Hom}_{S[G]}(M, N),
\end{array}
$$

is a functorial isomorphism of $R$-modules. The proof of this fact when the $S$-module $M$ is reflexive can be found in [Aus1, Proposition 2.2]. The general case reduces easily to this if one passes to a finite $S[G]$-free presentation of $M$. Thus $\left(v^{*}\right)^{G}$ is an isomorphism between the $R$-modules $\operatorname{Hom}_{R}\left(D_{k}(S)^{G}, R\right)$ and $\operatorname{Hom}_{R}\left(\left(D_{k}(R) \otimes_{R} S\right)^{G}, R\right)$. We now claim that $\left(D_{k}(R) \otimes_{R} S\right)^{G}$ is isomorphic to $D_{k}(R)$. This follows from a general fact: if $L$ is a finitely-generated $R$-module and $G$ acts on the $S$-module $L \otimes_{R} S$ via the second factor then the inclusion

$$
i_{L}: L \rightarrow\left(L \otimes_{R} S\right)^{G}: l \mapsto l \otimes 1, \quad l \in L,
$$

is a functorial isomorphism of $R$-modules. Indeed the functoriality of $i_{L}$ is obvious. Therefore, using a finite presentation of $L$ we can assume that $L$ is isomorphic to $R$. But $i_{R}$ is the fixed part of the $S[G]$-isomorphism $S \rightarrow$ $R \otimes_{R} S: s \rightarrow 1 \otimes s, s \in S$. Thus $\left(v^{*}\right)^{G}$ is an isomorphism of the $R$-modules $\left(D_{k}(S)^{G}\right)^{*}$ and $D_{k}(R)^{*}$. Since $D_{k}(S)$ is a reflexive $S$-module and the order of $G$ is invertible in $k, D_{k}(S)^{G}$ is a reflexive $R$-module. Therefore, $D_{k}(S)^{G} \cong$ $\left(D_{k}(S)^{G}\right)^{* *} \cong D_{k}(R)^{* *}$ and the proposition is proved.

Corollary. Let $S=k\left[\left|X_{1}, X_{2}\right|\right]$ be the formal power series ring in two variables over a field $k$ and $G$ a finite group of $k$-algebra automorphisms of $S$. Suppose that the order of $G$ is not divisible by the characteristic of $k$ and that $R:=S^{G}$ is unramified in $S$ at the height 1 primes (if $k$ is algebraically closed the last condition simply means that $G$ acts on the k-vector space $k X_{1} \amalg k X_{2}$ without pseudoreflections). Let $0 \rightarrow \omega_{R} \rightarrow A \rightarrow R \rightarrow k \rightarrow 0$ be the fundamental 
sequence (0.1) of $R$. Then the Auslander module $A$ is isomorphic to the module of Zariski differentials $D_{k}(R)^{* *}$ of $R$ over $k$.

Proof. Let $V$ be the $k$-vector space $k X_{1} \amalg k X_{2}$. As was shown in [Aus1, p. 516] the fundamental sequence is the fixed part of the $S[G]$-projective resolution of $k$ :

$$
0 \rightarrow S \otimes_{k} \Lambda^{2} V \stackrel{\left(\begin{array}{c}
-X_{2} \\
X_{1}
\end{array}\right)}{\longrightarrow} S \otimes_{k} V \stackrel{\left(X_{1} X_{2}\right)}{\longrightarrow} S \rightarrow k \rightarrow 0 .
$$

In particular $A \cong\left(S \otimes_{k} V\right)^{G}$. But $S \otimes_{k} V$ is $S[G]$-isomorphic to $D_{k}(S)$ under the map $1 \otimes X_{i} \rightarrow d X_{i}, i=1,2$. Hence $A \cong D_{k}(S)^{G} \cong D_{k}(R)^{* *}$ by Proposition 1.3.

\section{A GENERAL CRITERION AND A CONJECTURE}

Having established an isomorphism $A \cong D_{k}(R)^{* *}$ for quotient singularities we will now give a general criterion for the existence of such an isomorphism.

Lemma 2.1. Let $R$ be a complete two-dimensional integrally closed local domain. An $R$-module $M$ is isomorphic to the Auslander module $A$ of $R$ if and only if the following conditions are satisfied:

(a) $M$ is reflexive.

(b) $\operatorname{rank} M=2$,

(c) the divisor $c(M)$ of $M$ is the class $\left[\omega_{R}\right]$ of the dualizing module $\omega_{R}$ of $R$,

(d) $M$ can be mapped onto the maximal ideal $\mathfrak{m}$ of $R$.

Proof. "Only if". We already mentioned in the introduction that $A$ is reflexive, whence (a). The rest follows from (0.1).

"If". Suppose $p: M \rightarrow \mathfrak{m}$ is a surjection with kernel $K$. Composing $p$ with the inclusion $\mathfrak{m} \rightarrow R$ we can view $K$ as the kernel of a map between reflexive $R$-modules. Hence $K$ is reflexive. Obviously $\operatorname{rank} K=1$ and $c(K)=c(M)=$ $\left[\omega_{R}\right]$. Therefore $K$ is isomorphic to $\omega_{R}$. Since $\mathfrak{m}$ is not reflexive the sequence $0 \rightarrow \omega_{R} \rightarrow M \rightarrow \mathfrak{m} \rightarrow 0$ does not split and we obtain a nonsplit exact sequence $0 \rightarrow \omega_{R} \rightarrow M \rightarrow R \rightarrow R / \mathfrak{m} \rightarrow 0$. Since $\operatorname{Ext}_{R}^{2}(R / \mathfrak{m}, R) \cong R / \mathfrak{m}$ this sequence is isomorphic to the fundamental sequence $(0.1)$ and, therefore, $M$ is isomorphic to $A$.

We can now formulate our criterion.

Proposition 2.2. Let $R$ be a two-dimensional integrally closed analytic $k$-algebra over a perfect field $k$. Then the Auslander module $A$ of $R$ is isomorphic to the module $D_{k}(R)^{* *}$ of Zariski differentials if and only if there exists a surjection $D_{k}(R)^{* *} \rightarrow \mathfrak{m}$, where $\mathfrak{m}$ is the maximal ideal of $R$.

Proof. The "only if" part is obvious. For the converse we will show that $D_{k}(R)^{* *}$ satisfies conditions (a)-(d) of the previous lemma. The first condition is trivially satisfied. Since $R$ is a domain and $k$ is perfect, $\operatorname{rank} D_{k}(R)=$ 
$\operatorname{dim} R=2$ (see [SchSt, 4.2]), whence (b). Condition (a) is part of the hypothesis. Thus we only have to show that $c\left(D_{k}(R)^{* *}\right)=\left[\omega_{R}\right]$. This follows from a result of E. Platte $[\mathrm{P} 1,(6.4)]$, which says that $\omega_{R}$ is isomorphic to $\left(\bigwedge^{2} D_{k}(R)\right)^{* *}$ if $R$ is integrally closed and $D_{k}(R)$ is free at the height 1 primes. The last condition is satisfied by virtue of the jacobian criterion (see [Sch, Theorem 25]) which says that if $k$ is perfect and $R$ is reduced then for any prime ideal $q$ of $R$ the module $D_{k}(R)_{q}$ is free if and only if $R_{q}$ is regular. Therefore $c\left(D_{k}(R)^{* *}\right)=c\left(\left(\bigwedge^{2} D_{k}(R)^{* *}\right)=c\left(\omega_{R}\right)=\left[\omega_{R}\right]\right.$. The proposition is proved.

This result has an immediate application. Recall [SchW1, p. 10] that an analytic $k$-algebra over a field $k$ of characteristic 0 is called (positively) graded if there exists a $k$-derivation $\delta: R \rightarrow R$ and a system of generators $x_{1}, \ldots, x_{d}$ of the maximal ideal $\mathfrak{m}$ of $R$ such that $\delta x_{i}=m_{i} x_{i}$, where the $m_{i}$ 's are positive integers, $i=1, \ldots, d$.

Remark. This definition of a grading extends the usual definition to the class of complete (or convergent) power series rings and their quotients (see [SchW2, p. 166]). Using [ibid, 2.1, p. 167] and the proof of [ibid, Satz 2.2, p. 168] one can easily show that a graded (complete) analytic $k$-algebra is just the completion of a graded (in the usual sense) quotient of a polynomial algebra.

Corollary 2.3. Suppose $R$ is a nonregular integrally closed graded Gorenstein analytic k-algebra of Krull dimension 2. Then the Auslander module $A$ of $R$ is isomorphic to the module of Zariski differentials $D_{k}(R)^{* *}$.

Proof. The grading derivation $\delta: R \rightarrow R$ gives rise to a homomorphism $h$ : $D_{k}(R) \rightarrow R$ whose image is the maximal ideal $\mathfrak{m}$ of $R$. We claim that the homomorphism $h^{* *}: D_{k}(R)^{* *} \rightarrow R$ also has $\mathfrak{m}$ as its image. If it were not the case, $h^{* *}$ would be a split epimorphism and we would have a direct sum decomposition $D_{k}(R)^{* *} \cong K \amalg R$. Since $K$ is a rank 1 reflexive and $c(K)=$ $\left[\omega_{R}\right], K$ is isomorphic to $\omega_{R}$. Since $R$ is Gorenstein, $\omega_{R}$ is isomorphic to $R$, which makes $D_{k}(R)^{* *}$ a free module. The Zariski-Lipman conjecture, established in the graded case [Ho, $\mathrm{P} 2]$, implies then that $R$ is regular, contrary to the hypothesis. Thus we have a surjection $h^{* *}: D_{k}(R)^{* *} \rightarrow \mathfrak{m}$. Since $R$ is graded, $k$ is, by definition, of characteristic 0 . The desired result follows from Proposition 2.2.

We now have two classes of analytic $k$-algebras for which there exists an isomorphism $A \cong D_{k}(R)^{* *}$, namely, quotient singularities and graded Gorenstein analytic $k$-algebras. If $G$ is a finite subgroup of $\operatorname{GL}(2, \mathbf{C})$ which is not a subgroup of $\mathrm{SL}(2, \mathrm{C})$ then the corresponding quotient singularity is not Gorenstein. Thus $R$ need not be Gorenstein in order for the modules $A$ and $D_{k}(R)^{* *}$ to be isomorphic. On the other hand, quotient singularities are graded. These simple observations point in the direction of

Conjecture 2.4. Let $R$ be a two-dimensional integrally closed analytic $k$-algebra over a field $k$ of characteristic 0 . Let $0 \rightarrow \omega_{R} \rightarrow A \rightarrow R \rightarrow R / \mathfrak{m} \rightarrow 0$ be 
the fundamental exact sequence (i.e., a representative of a nonzero element of $\left.\operatorname{Ext}_{R}^{2}(R / \mathfrak{m}, R)\right)$. Then the Auslander module $A$ is isomorphic to the module $D_{k}(R)^{* *}$ of Zariski differentials if and only if $R$ is graded.

The main result of this paper is a proof of this conjecture for hypersurface singularities $f=X_{3}^{n}+g\left(X_{1}, X_{2}\right)$ (see Theorem 3.2 below). Additional evidence supporting this conjecture is gathered in the following remarks:

Remarks. 1. J. Wahl pointed out that the "if" part of Conjecture 2.4 holds in the complex-analytic category. This follows from a result of Naruki [Nar, 2.1] (see [Wahl, (1.2)]) and Proposition 2.2.

2. K. Behnke kindly informed us that Conjecture 2.4 holds for (a) minimally elliptic singularities, and (b) rational singularities with reduced fundamental cycle.

\section{The MAIN THEOREM}

We begin by recalling the following result (first proved by K. Saito [Sai] for $k=\mathbf{C}$ ) which characterizes graded isolated hypersurface singularities in terms of the jacobian ideal of the defining power series.

Proposition 3.1 (see [SchW2, Satz, 5.7, p. 183]). Let $T$ be the formal power series ring in variables $X_{1}, \ldots, X_{n}, n \geq 2$, over an algebraically closed field $k$ of characteristic 0 . Let $f$ be a formal power series whose jacobian ideal $j(f)$ is $\mathfrak{m}_{T}$-primary. Then the following are equivalent:

(1) $f \in j(f)$.

(2) $T /(f)$ is positively graded.

(3) There is a positive grading of $T$ in which $f$ is a homogeneous polynomial.

Remark. This result is proved in [SchW2] in greater generality and contains several more equivalent conditions. We only quoted what is needed for our purposes.

We can now state our main result.

Theorem 3.2. Let $S=k\left[\left|X_{1}, X_{2}, X_{3}\right|\right]$ be the formal power series ring over an algebraically closed field $k$ of characteristic 0 . Let $f:=X_{3}^{n}+g\left(X_{1}, X_{2}\right) \in \mathfrak{m}_{S}$ be a formal power series whose jacobian ideal $j(f)=\left(\partial f / \partial X_{1}, \partial f / \partial X_{2}, \partial f / \partial X_{3}\right)$ is $\mathfrak{m}_{S}$-primary (this is equivalent to the jacobian ideal of $g$ being $\left(X_{1}, X_{2}\right)$ primary). Let $R:=S /(f)$ and $0 \rightarrow \omega_{R} \rightarrow A \rightarrow R \rightarrow k \rightarrow 0$ be the fundamental sequence. Then the Auslander module $A$ is isomorphic to the mo:dule $D_{k}(R)^{* *}$ of Zariski differentials of $R$ over $k$ if and only if $f \in j(f)$.

To prove this theorem we will compare presentation matrices of $A$ and $D_{k}(R)^{* *}$. Since $R$ is an integrally closed domain (Serre's criterion) of dimension 2 both modules are Cohen-Macaulay (C-M for short). By a result of Eisenbud [Eis, Corollary 6.3], C-M modules without free summands over the hypersurface ring $R$ are reductions modulo $(f)$ of reduced matrix factorizations of $f$. Recall [ibid] that a matrix factorization of $f$ is an ordered pair of 
homomorphisms $\left(\varphi: S^{k} \rightarrow S^{k}, \psi: S^{k} \rightarrow S^{k}\right)$ such that $\varphi \psi=\psi \varphi=f \cdot \operatorname{Id}_{S^{k}}$. A matrix factorization $(\varphi, \psi)$ is called reduced if the matrix elements of $\varphi$ and $\psi$ belong to the maximal ideal $\mathfrak{m}_{S}$ of the local ring $S$. It is easy to see that $\operatorname{Coker} \varphi$ is an $R$-module. Moreover, reducing $\varphi$ and $\psi$ modulo $(f)$, we obtain a minimal 2-periodic resolution of the $R$-module $\operatorname{Coker} \varphi$ (and, interchanging $\varphi$ and $\psi$, of $\operatorname{Coker} \psi$ ). If $f$ is a prime and $\operatorname{det} \varphi=f^{r}$ then $r=\operatorname{rank}_{R} \operatorname{Coker} \varphi$ [ibid, Corollary 6.4]. Thus we can use matrix factorizations to describe the periodic parts of projective resolutions over $R$. Later we will see that both $A$ and $D_{k}(R)^{* *}$ come as higher syzygy modules of certain modules of finite length. To show this we will have to describe the beginning of a projective resolution. As it often happens the problem is not to find relations but to prove that we found all of them. An acyclicity lemma proved in the next section will enable us to do this.

\section{AN ACYCLICITY LEMMA AND APPLICATIONS}

Lemma 4.1. Let $R$ be a commutative noetherian integrally closed local domain and

$$
\mathbf{M}: 0 \rightarrow M_{2} \stackrel{\varphi_{2}}{\longrightarrow} M_{1} \stackrel{\varphi_{1}}{\longrightarrow} M_{0} \rightarrow 0
$$

a complex of finitely generated torsion-free modules. Suppose that

(a) $\mathbf{M}$ is exact except, possibly, at $M_{1}$,

(b) $\operatorname{rank} M_{1}=\operatorname{rank} M_{2}+\operatorname{rank} M_{0}$,

(c) $\operatorname{Im} \varphi_{2}$ and $\operatorname{Ker} \varphi_{1}$ are reflexive,

(d) the localization of $\varphi_{2}$ at every height 1 prime of $R$ is split.

Then $\mathbf{M}$ is exact.

Proof. We want to show that the natural inclusion $i: \operatorname{Im} \varphi_{2} \rightarrow \operatorname{Ker} \varphi_{1}$ is an isomorphism. Since both modules are reflexive it suffices to show that the localization of $i$ at every height 1 prime of $R$ is an isomorphism. Thus we can assume that $\mathbf{M}$ is a complex of free modules. But then the natural map Coker $\varphi_{2} \rightarrow M_{0}$ can be viewed as a surjective endomorphism of a noetherian module. Therefore it is an isomorphism and the result follows.

Remark. The proof shows that the assertion of Lemma 4.1 remains true if we assume that the modules $M_{i}$ have torsion submodules pseudoisomorphic to 0 .

Proposition 4.2. Let $S$ be a regular local ring of dimension $3, \mathfrak{m}_{S}$ the maximal ideal of $S$ and $f \in \mathfrak{m}_{S}^{2}$. Let $f=g_{1} t_{1}+g_{2} t_{2}+g_{3} t_{3}$, where $t_{1}, t_{2}, t_{3}$ is a system of parameters of $S$ and $\left(g_{1}, g_{2}, g_{3}\right) \subset \mathfrak{m}_{S}$. Suppose that $R:=S /(f)$ is an integrally closed domain (in particular $f$ is a prime). Then the complex

$$
\cdots \stackrel{\bar{\Psi}}{\longrightarrow} R^{4} \stackrel{\bar{\Phi}}{\longrightarrow} R^{4} \stackrel{\bar{\Psi}}{\longrightarrow} R^{4} \stackrel{\bar{\Sigma}}{\longrightarrow} R^{3} \stackrel{\bar{T}}{\longrightarrow} R \rightarrow R /\left(\bar{t}_{1}, \bar{t}_{2}, \bar{t}_{3}\right) \rightarrow 0,
$$

where

$$
\Phi:=\left(\begin{array}{rrrr}
g_{3} & g_{2} & g_{1} & 0 \\
t_{2} & -t_{3} & 0 & g_{1} \\
-t_{1} & 0 & t_{3} & g_{2} \\
0 & t_{1} & -t_{2} & g_{3}
\end{array}\right) \text {, }
$$




$$
\begin{aligned}
\Psi: & =\left(\begin{array}{rrrr}
t_{3} & g_{2} & -g_{1} & 0 \\
t_{2} & -g_{3} & 0 & g_{1} \\
t_{1} & 0 & g_{3} & -g_{2} \\
0 & t_{1} & t_{2} & t_{3}
\end{array}\right), \\
\Sigma & =\left(\begin{array}{rrrr}
t_{2} & -t_{3} & 0 & g_{1} \\
-t_{1} & 0 & t_{3} & g_{2} \\
0 & t_{1} & -t_{2} & g_{3}
\end{array}\right),
\end{aligned}
$$

$T:=\left(t_{1} t_{2} t_{3}\right)$ and the overbars denote reduction modulo $(f)$, is a minimal projective resolution of $R /\left(\bar{t}_{1}, \bar{t}_{2}, \bar{t}_{3}\right)$.

Proof. First we notice that $\Psi \Phi=\Phi \Psi=f \cdot \operatorname{Id}_{S^{4}}$. Thus $(\Phi, \Psi)$ is a matrix factorization of $f$ and, therefore, $(*)$ is exact in degrees $>2$. It remains to prove the exactness in degrees 1 and 2 . To show that $\bar{\Sigma}$ gives a basis of relations between $\bar{t}_{1}, \bar{t}_{2}$ and $\bar{t}_{3}$ we consider an arbitrary relation $\bar{r}_{1} \bar{t}_{1}+\bar{r}_{2} \bar{t}_{2}+\bar{r}_{3} \bar{t}_{3}=0$, where $\bar{r}_{i} \in R, i=1,2,3$. Lifting each $\bar{r}_{i}$ in an arbitrary way to an element $r_{i} \in S$ we have $r_{1} t_{1}+r_{2} t_{2}+r_{3} t_{3}=a f$ for some $a \in S$. Subtracting from this relation the relation $a g_{1} t_{1}+a g_{2} t_{2}+a g_{3} t_{3}=a f$ we obtain a relation between $t_{1}, t_{2}$ and $t_{3}$ in $S$. Since the $t_{i}$ 's form a system of parameters, all such relations are generated by the Koszul relations. Therefore, the module of relations over $R$ between $\bar{t}_{1}, \bar{t}_{2}$ and $\bar{t}_{3}$ is generated by the images of the Koszul relations and the image of $g_{1} t_{1}+g_{2} t_{2}+g_{3} t_{3}=f$. This shows that $(*)$ is exact in degree 1 .

To show that $(*)$ is exact in degree 2 is equivalent to showing that the complex

$$
0 \rightarrow \operatorname{Im} \bar{\Psi} \stackrel{\varphi_{2}}{\rightarrow} R^{4} \stackrel{\varphi_{1}}{\rightarrow} \operatorname{Im} \bar{\Sigma} \rightarrow 0
$$

is exact, where $\varphi_{2}$ is the natural inclusion and $\varphi_{1}$ is the natural surjection. We invoke the acyclicity lemma. It is clear that all the modules in $(* *)$ are torsionfree. By construction this complex is exact except, possibly, in the middle. Next we check that $\operatorname{rank} \operatorname{Im} \bar{\Psi}+\operatorname{rank} \operatorname{Im} \bar{\Sigma}=4$. Since $(*)$ is exact in degree 1 we have $\operatorname{rank} \operatorname{Im} \bar{\Sigma}=2$. On the other hand, $\operatorname{Im} \bar{\Psi}$ is isomorphic to $\operatorname{Coker} \bar{\Phi}$ because $(\Phi, \Psi)$ is a matrix factorization. Since $\operatorname{det} \Psi=f^{2}$ and $f$ is a prime rank Coker $\bar{\Phi}=2$. Thus $(* *)$ has correct ranks. Now we want to check that $\operatorname{Im} \varphi_{2}$ and $\operatorname{Ker} \varphi_{1}$ are reflexivie. But $\operatorname{Im} \varphi_{2} \cong \operatorname{Im} \bar{\Psi} \cong \operatorname{Coker} \bar{\Phi}$ is C-M and, therefore, reflexive, and $\operatorname{Ker} \varphi_{1} \cong \operatorname{Ker} \bar{\Sigma}$ is reflexive as the kernel of a map between free modules. Finally we check that $\operatorname{Im} \varphi_{2}=\operatorname{Im} \bar{\Psi}$ is a direct summand of $R^{4}$ at the height 1 primes of $R$. The matrix factorization $(\Phi, \Psi)$ gives rise to the exact sequence $R^{4} \stackrel{\bar{\Psi}}{\rightarrow} R^{4} \stackrel{\bar{\Phi}}{\rightarrow} R^{4}$ and, therefore, to the exact sequence

$$
0 \rightarrow \operatorname{Im} \bar{\Psi} \stackrel{\varphi_{2}}{\rightarrow} R^{4} \rightarrow \operatorname{Im} \bar{\Phi} \cong \operatorname{Coker} \bar{\Psi} \rightarrow 0 .
$$

Since $(\Psi, \Phi)$ is also a matrix factorization $\operatorname{Coker} \bar{\Psi}$ is reflexive and, therefore, $\varphi_{2}$ splits at each height 1 prime of $R$. The desired result now follows from the acyclicity lemma.

Corollary 4.3. The cokernel of $\bar{\Phi}$ can be mapped onto the ideal $\left(\bar{t}_{1}, \bar{t}_{2}, \bar{t}_{3}\right)$ of $R$. 
Proof. Let $B$ denote the matrix

$$
\left(\begin{array}{llll}
0 & 1 & 0 & 0 \\
0 & 0 & 1 & 0 \\
0 & 0 & 0 & 1
\end{array}\right)
$$

Then $(*)$ gives rise to the commutative diagram

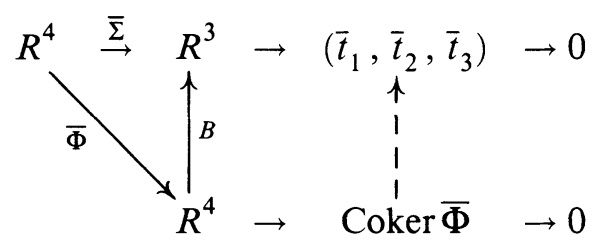

which proves our assertion.

If we replace $t_{1}, t_{2}, t_{3}$ with a system of generators $X_{1}, X_{2}, X_{3}$ of the maximal ideal $\mathrm{m}_{S}$ of $S$ and write $f=f_{1} X_{1}+f_{2} X_{2}+f_{3} X_{3}$ we will see that Coker $\bar{\Phi}$ is a rank 2 reflexive module with trivial divisor and that it can be mapped onto the maximal ideal $\left(\bar{X}_{1}, \bar{X}_{2}, \bar{X}_{3}\right)$ of $R$. By Lemma 2.1 these are the characteristic properties of the Auslander module $A$ of $R$. Thus $\bar{\Phi}$ is a presentation matrix for $A$ and $\bar{\Psi}$ is a presentation matrix for the first syzygy module of $A$. We write down these matrices again for further references

$$
\begin{aligned}
\Phi & =\left(\begin{array}{rrrr}
f_{3} & f_{2} & f_{1} & 0 \\
X_{2} & -X_{3} & 0 & f_{1} \\
-X_{1} & 0 & X_{3} & f_{2} \\
0 & X_{1} & -X_{2} & f_{3}
\end{array}\right), \\
\Psi & =\left(\begin{array}{rrrr}
X_{3} & f_{2} & -f_{1} & 0 \\
X_{2} & -f_{3} & 0 & f_{1} \\
X_{1} & 0 & f_{3} & -f_{2} \\
0 & X_{1} & X_{2} & X_{3}
\end{array}\right),
\end{aligned}
$$

where $f=f_{1} X_{1}+f_{2} X_{2}+f_{3} X_{3}$.

Remark. The resolution of the residue field of an arbitrary hypersurface ring was first obtained by J. Tate [Tate]. Our approach, although restricted to integrally closed hypersurface rings of dimension 2 , is more elementary.

\section{ZARISKI DIFFERENTIALS OF TWO-DIMENSIONAL ISOLATED HYPERSURFACE SINGULARITIES}

Lemma 5.1. Let $\left(S, \mathfrak{m}_{S}\right)$ be a regular local ring of dimension $3, f \in m_{S}$ and $R:=S /(f)$. Let $f_{1}, f_{2}, f_{3}$ be a system of parameters of $S$ generating an ideal a $\subset S$. Let $M$ be the $R$-module defined by the exact sequence

$$
0 \rightarrow R \stackrel{\left(\begin{array}{l}
\bar{f}_{1} \\
\bar{f}_{2} \\
\bar{f}_{3}
\end{array}\right)}{\rightarrow} R^{3} \rightarrow M \rightarrow 0 .
$$


Then

(a) there exists an exact sequence

$$
0 \rightarrow M \rightarrow M^{*} \rightarrow(\mathfrak{a}:(f)) / \mathfrak{a} \rightarrow 0 .
$$

(b) the module $M^{*}$ is self-dual, i.e., $M^{* *} \cong M^{*}$.

Proof. The second assertion follows obviously from the first. To prove (a) we calculate $\operatorname{Tor}_{i}^{S}(S /(f), S / \mathfrak{a})$. On one hand, tensoring $0 \rightarrow S \stackrel{\cdot f}{\rightarrow} S \rightarrow S /(f) \rightarrow 0$ with $S / \mathfrak{a}$ we see that $\operatorname{Tor}_{1}^{S}(S /(f), S / \mathfrak{a}) \cong(\mathfrak{a}:(f)) / \mathfrak{a}$ and $\operatorname{Tor}_{i}^{S}(S /(f), S / \mathfrak{a})=0$ for $i>1$. On the other hand we have the Koszul resolution (over $S$ ) of $S / \mathfrak{a}$ : $0 \rightarrow S \rightarrow S^{3} \rightarrow S^{3} \rightarrow S \rightarrow S / \mathfrak{a} \rightarrow 0$. Tensoring it with $S /(f)$ we obtain an augmented complex whose only nonzero homology is in degree 1 and is equal to $\operatorname{Tor}_{1}^{S}(S /(f), S / \mathfrak{a})$ :

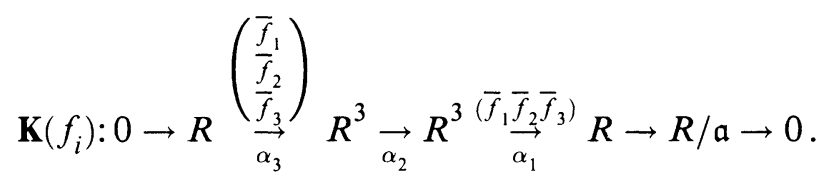

We now see that $M$ is isomorphic to $\operatorname{Coker} \alpha_{3}$ and $M^{*}$ is isomorphic to $\operatorname{Ker} \alpha_{1}$. Since $\mathbf{K}\left(\bar{f}_{i}\right)$ is exact in degree 2, Coker $\alpha_{3} \cong \operatorname{Im} \alpha_{2}$. Therefore $(\mathfrak{a}:(f)) / \mathfrak{a} \cong \operatorname{Tor}_{1}^{S}(S /(f), S / \mathfrak{a}) \cong \operatorname{Ker} \alpha_{1} / \operatorname{Im} \alpha_{2}$, which proves (a).

Corollary 5.2. Suppose $R=S /(f)$, where $S=k\left[\left|X_{1}, X_{2}, X_{3}\right|\right]$ is a formal power series ring over a field $k$ and $f \in \mathfrak{m}_{S}$. Suppose that the jacobian ideal $j(f)$ is $\mathfrak{m}_{S}$-primary. Then the module of Zariski differentials $D_{k}(R)^{* *}$ is isomorphic to the module of derivations $D_{k}(R)^{*}$.

Proof. In the previous lemma take $M:=D_{k}(R)$ and $f_{i}:=\partial f / \partial X_{i}, i=$ $1,2,3$ :

$$
0 \rightarrow R^{\left(\frac{\frac{\partial f / \partial X_{1}}{\partial f / \partial X_{2}}}{\partial f / \partial X_{3}}\right)} R^{3} \rightarrow D_{k}(R) \rightarrow 0
$$

Dualizing this projective resolution of $D_{k}(R)$ we obtain

Corollary 5.3. The module of Zariski differentials of a two-dimensinal isolated hypersurface singularity $R:=S /(f)$ is the second syzygy module of the finitedimensional algebra $R / \overline{j(f)}$.

This finite-dimensional algebra is sometimes called the moduli algebra of $f$. Thus to obtain a presentation of the module of Zariski differentials $D_{k}(R)^{* *}$ of the isolated hypersurface singularity $R$ we have to find a projective resolution of the moduli algebra $R / \overline{j(f)}$. This problem remains unsolved in general (and even in our case of three variables). However in the case of two variables we were able to obtain a satisfactory solution which will later enable us to solve the same problem for two-dimensional hypersurfaces $f:=X_{3}^{n}+g\left(X_{1}, X_{2}\right)$. 


\section{A MiNimAl RESOlUtion OF THE MODUli ALGEBRA OF A FORMAL PLANE CURVE WITH AN ISOLATED SINGULARITY}

Lemma 6.1. Let $\left(S, \mathfrak{m}_{S}\right)$ be a formal power series ring in $X_{1}, \ldots, X_{n}$ over a field $k$ of characteristic 0 . Let $g \in S$ be a formal power series with $\mathfrak{m}_{S}$-primary jacobian ideal $j(g)$. Then the partial derivatives of $g$ are part of a minimal system of generators of the ideal $(g, j(g)) \subset S$.

Proof. This is any easy consequence of a result of Zariski (see [Te, p. 586]). Suppose the assertion is false and we have a relation, say $\partial g / \partial X_{n}=a g+$ $\sum_{1}^{n-1} b_{i}\left(\partial g / \partial X_{i}\right)$, where $a, b_{i} \in S, i=1, \ldots, n-1$. Consider the $k$ derivation $\delta:=\partial / \partial X_{n}-\sum_{1}^{n-1} b_{i}\left(\partial / \partial X_{i}\right)$ of $S$ into itself. Then $\delta g=a g$ and $\delta$ gives rise to a $k$-derivation $\bar{\delta}$ of $R:=S /(g)$ into itself. Notice that $\bar{\delta}\left(\bar{X}_{n}\right)=1$, i.e., the image of $\bar{\delta}$ contains a unit. By the aforementioned result of Zariski there exist $z \in R$ and a subring $R_{1}$ of $R$ containing $k$ such that $R=R_{1}[|z|]$. If $R_{1}$ were regular then $R$ would be regular and by the jacobian criterion the module $D_{k}(R)$ of Kähler differentials would be free contrary to the assumption that $j(g)$ is $\mathfrak{m}_{S}$-primary. Thus $R_{1}$ is not regular. But then $R=R_{1}[|z|]$ is not an isolated singularity contrary to the assumption that $j(g)$ is $\mathfrak{m}_{S}$-primary. The lemma is proved.

Remark. The proof shows that no partial derivative of $g$ is an $S$-linear combination of $g$ and other partial derivatives.

We now specialize to the case $n=2$. Our goal is to obtain a minimal resolution over $R$ of $R / \overline{j(g)}$. This resolution can be produced in a very general setting once we postulate the properties of the partials mentioned in the above remark. To this end we replace $k\left[\left|X_{1}, X_{2}\right|\right]$ with an arbitrary regular local ring $U$ of Krull dimension 2 with the maximal ideal $\mathfrak{m}_{U}$. Suppose we are given a system of parameters $g_{1}, g_{2} \in \mathfrak{m}_{U}$ (the "analogues" of the partials) generating an ideal $j \subset U$, and an element $g \in U$. Suppose furthermore that $g_{1}$ (resp. $g_{2}$ ) is not a $U$-linear combination of $g$ and $g_{2}$ (resp. $g_{1}$ ). In particular $g_{1}$ and $g_{2}$ are part of a minimal generating set of the ideal $(g, j)=\left(g, g_{1}, g_{2}\right)$. Let $V:=U /(g)$ and $\bar{g}_{1}, \bar{g}_{2}$ and $\bar{j}$ be the images of $g_{1}, g_{2}$ and $j$ in $V$. We will describe a minimal projective resolution over $V$ of $V /\left(\bar{g}_{1}, \bar{g}_{2}\right)=V / \bar{j}$.

First we treat the case $g \in j$. If $g=a_{1} g_{1}+a_{2} g_{2}$, where $a_{1}, a_{2} \in U$, then, because of the above assumption on $g_{1}$ and $g_{2}$, the elements $a_{1}$ and $a_{2}$ belong to $\mathfrak{m}_{U}$. The relations between $g, g_{1}$ and $g_{2}$ (over $U$ ) and generated by $g=a_{1} g_{1}+a_{2} g_{2}$ and the Koszul relations on $g_{1}$ and $g_{2}$. Using matrix factorization of $g$ we obtain the following (periodic in degree $\geq 1$ ) minimal resolution over $V$ :

$$
\cdots \rightarrow V^{2} \stackrel{\left(\begin{array}{cc}
\bar{g}_{1} & \bar{g}_{2} \\
-\bar{a}_{2} & \bar{a}_{1}
\end{array}\right)}{\stackrel{a_{1}}{\longrightarrow}} V^{2} \stackrel{\left(\begin{array}{cc}
\bar{a}_{1} & -\bar{g}_{2} \\
\bar{a}_{2} & \bar{g}_{1}
\end{array}\right)}{\rightarrow} V^{2\left(\bar{g}_{1} \bar{g}_{2}\right)} V \rightarrow V / \bar{j} \rightarrow 0 .
$$

This finishes the case $g \in j$. 
We now assume that $g \notin j$. Therefore $g, g_{1}$ and $g_{2}$ are subject to the condition

$$
\begin{aligned}
& g, g_{1} \text { and } g_{2} \text { form a minimal generating set of the ideal } \\
& \left(g, g_{1}, g_{2}\right) \subset U .
\end{aligned}
$$

Hence $\left(g, g_{1}, g_{2}\right)$ is an almost complete intersection.

Lemma 6.2. The ideal $j:(g) \subset U$ is minimally generated by two elements.

Proof. Since height $(j:(g))=2$ it suffices to prove that $j:(g)$ is a Gorenstein ideal (i.e., $U /(j:(g))$ is Gorenstein). Indeed, by a result of Serre [Se, Proposition 3], a Gorenstein ideal of height 2 of a regular local ring can be generated two elements. To show that $j:(g)$ is a Gorenstein ideal we invoke the notion of linkage, the definition of which we recall [PS].

Definition. Let $I$ and $J$ be ideals of a Macaulay ring $R$ of pure height (= grade) $h$. We say that $I$ is linked to $J$ if there is an $R$-sequence $x_{1}, \ldots, x_{n} \in I \cap J$ such that $J=\left(x_{1}, \ldots, x_{n}\right): I$.

Having defined the linkage we immediately verify that $\left(g, g_{1}, g_{2}\right)$ is linked to $j:(g)$ by the sequence $g_{1}, g_{2}$. Notice that both ideals $\left(g, g_{1}, g_{2}\right)$ and $j:(g)$ are perfect of the same grade 2 and, as we already mentioned, $\left(g, g_{1}, g_{2}\right)$ is an almost complete intersection. The desired result now follows from a theorem of Buchsbaum-Eisenbud:

Proposition [BE, Proposition 5.2]. Let $I$ and $J$ be perfect ideals of the same grade $h$ in a Gorenstein local ring $R$, and suppose that $I$ is linked to $J$ by an R-sequence $x_{1}, \ldots, x_{n}$. Then

(1) If $J$ is Gorenstein, then $I$ is an almost complete intersection.

(2) If $I$ is an almost complete intersection and $x_{1}, \ldots, x_{n}$ form part of a minimal set of generators for $I$, then $J$ is Gorenstein.

This finishes the proof of Lemma 6.2.

Now let $b_{1}$ and $b_{2}$ be minimal generators of the ideal $j:(g)$. Then there exist $a_{i j} \in U, i, j=1,2$, such that

$$
\left\{\begin{array}{l}
a_{11} g_{1}+a_{21} g_{2}=b_{1} g \\
a_{12} g_{1}+a_{22} g_{2}=b_{2} g
\end{array}\right.
$$

or, in the matrix form,

$$
\left(\begin{array}{ll}
a_{11} & a_{21} \\
a_{12} & a_{22}
\end{array}\right)\left(\begin{array}{l}
g_{1} \\
g_{2}
\end{array}\right)=\left(\begin{array}{l}
b_{1} g \\
b_{2} g
\end{array}\right) .
$$

Condition (M) implies that $a_{i j} \in \mathfrak{m}_{U}, i, j=1,2$. 
Now we turn our attention to the ring $V=U /(g)$. Because of condition (M) we can start a minimal resolution of $V /\left(\bar{g}_{1}, \bar{g}_{2}\right)$ over $V$ as follows:

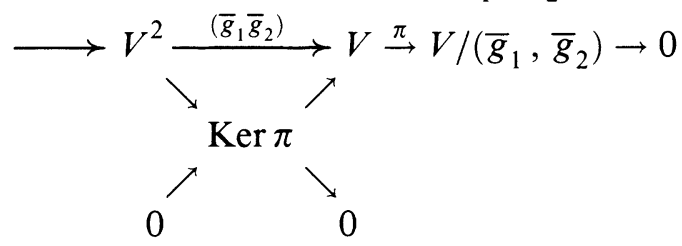

Since $V$ is a C-M ring of dimension 1 the first syzygy module $\operatorname{Ker} \pi$ is CM. We claim that $\operatorname{Ker} \pi$ has no free summands. Suppose it is not true and $\operatorname{Ker} \pi \cong I_{1} \amalg I_{2}$, where $I_{2} \cong V$. Since $\operatorname{Ker} \pi$ is an ideal, $I_{1}$ and $I_{2}$ are ideals too. And since $I_{1} \cdot I_{2} \subset I_{1} \cap I_{2}=\{0\}$, the ideal $I_{2}$ is annihilated by the ideal $I_{1}$. Hence $I_{2}$ consists entirely of torsion elements. But this is impossible if $I_{2} \cong V$.

Thus $\operatorname{Ker} \pi$ is a C-M module without free summands over the hypersurface ring $V=U /(g)$. Hence its minimal resolution is periodic of period (at most) 2. Hence (6.3) can be extended to

$$
\cdots \stackrel{\bar{\sigma}}{\rightarrow} V^{2} \stackrel{\bar{\tau}}{\rightarrow} V^{2} \stackrel{\bar{\sigma}}{\rightarrow} V^{2} \stackrel{\left(\bar{g}_{1} \bar{g}_{2}\right)}{\rightarrow} V \stackrel{\pi}{\rightarrow} V /\left(g_{1}, g_{2}\right) \rightarrow 0,
$$

where $(\sigma, \tau)$ is a matrix factorization of $g$ corresponding to the $V$-module $\operatorname{Ker} \pi$. The columns of $\bar{\sigma}$ are reductions modulo $(g)$ of relations (over $U$ ) between $g, g_{1}$ and $g_{2}$. Since $j:(g)=\left(b_{1}, b_{2}\right)$ and since $g_{1}, g_{2}$ is a system of parameters, all such relations are generated by the equations (6.1) and the Koszul relation on $g_{1}$ and $g_{2}$ :

$$
\left\{\begin{array}{l}
a_{11} g_{1}+a_{21} g_{2}=b_{1} g, \\
a_{12} g_{1}+a_{22} g_{2}=b_{2} g, \\
g_{2} g_{1}+\left(-g_{1}\right) g_{2}=0 .
\end{array}\right.
$$

We claim that the Koszul relation is a linear combination of the first two relations. To this end consider a minimal $U$-projective resolution of the module of finite length $N:=U /\left(g, g_{1}, g_{2}\right)$. Since $U$ is regular local $N$ has finite projective dimension and by the theorem of Auslander-Buchsbaum-Serre, p.d. $N=\operatorname{dim} U-\operatorname{depth} N=2$. Hence the resolution looks as follows:

$$
0 \rightarrow U^{n_{2}} \rightarrow U^{n_{1}} \rightarrow U \rightarrow U /\left(g, g_{1}, g_{2}\right) \rightarrow 0 \text {. }
$$

Since $g, g_{1}$ and $g_{2}$ are minimal generators (condition (M)!) $n_{1}=3$ and, therefore, $n_{2}=2$. Thus one of the relations (6.5) is a linear combination of the other two. It cannot be the first one, since then we would have $b_{1} \in\left(b_{2}\right)$, contrary to the fact that $j:(g)=\left(b_{1}, b_{2}\right)$ is minimally generated by two elements. The same argument applies to the second relation. Thus the Koszul relation must be a linear combination of relations (6.1) and we conclude that

$$
\sigma=\left(\begin{array}{ll}
a_{11} & a_{12} \\
a_{21} & a_{22}
\end{array}\right) .
$$

The claim just proved gives us even more information about $\sigma$. Suppose that the third relation in (6.5) is a linear combination of the first two with coefficients 
$d_{1}$ and $d_{2}$. Then $d_{1} b_{1}+d_{2} b_{2}=0$. Since $b_{1}$ and $b_{2}$ are relatively prime (otherwise they would belong to a principal ideal and could not generate the $\mathfrak{m}_{U}$-primary ideal $\left.j:(g)\right)$ we have $d_{1}=b_{2} \chi$ and $d_{2}=-b_{1} \chi$ for some $\chi \in U$. Since $\chi$ divides the coefficients $g_{2}$ and $\left(-g_{1}\right)$ in the Koszul relation, $\chi$ must be a unit. Replacing the generators $b_{1}$ and $b_{2}$ of $j:(g)$ with $b_{1} \chi$ and $b_{2} \chi$ we may assume that $\chi=1$. From the linear dependence of the relations $(6.5)$ we obtain

$$
\left\{\begin{array}{l}
g_{1}=-b_{1} a_{21}+b_{1} a_{22} \\
g_{2}=b_{2} a_{11}-b_{1} a_{12}
\end{array}\right.
$$

or, in the matrix form,

$$
\left(\begin{array}{l}
g_{1} \\
g_{2}
\end{array}\right)=\left(\begin{array}{cc}
a_{22} & -a_{21} \\
-a_{12} & a_{11}
\end{array}\right)\left(\begin{array}{l}
b_{1} \\
b_{2}
\end{array}\right) .
$$

Multiplying both sides of (6.7) by the transpose of $\sigma$ on the left we have, in view of $(6.2)$ :

$$
\left(\begin{array}{l}
b_{1} g \\
b_{2} g
\end{array}\right)=\left(\begin{array}{l}
\operatorname{det} \alpha \cdot b_{1} \\
\operatorname{det} \alpha \cdot b_{2}
\end{array}\right),
$$

whence $\operatorname{det} \sigma=g$. It now follows that

$$
\sigma=\left(\begin{array}{ll}
a_{11} & a_{12} \\
a_{21} & a_{22}
\end{array}\right), \quad \tau=\left(\begin{array}{cc}
a_{22} & a_{12} \\
-a_{21} & a_{11}
\end{array}\right)
$$

and

$$
\operatorname{det} \tau=\operatorname{det} \sigma=g .
$$

This yields the desired resolution of $V /\left(\bar{g}_{1}, \bar{g}_{2}\right)$.

\section{A minimal Resolution of the MOdUli AlgebRa OF $f=X_{3}^{n}+g\left(X_{1}, X_{2}\right)$}

Let $S$ be the formal power series ring in variables $X_{1}, X_{2}$ and $X_{3}$ over a field $k$ of characteristic 0 . Suppose $f=X_{3}^{n}+g\left(X_{1}, X_{2}\right) \in \mathfrak{m}_{S}$ is a formal power series whose jacobian ideal $j(f)$ is $\mathfrak{m}_{S}$-primary (in particular, $n \geq 2$ ). Let $R:=S /(f)$. By Serre's criterion, $R$ is an integrally closed domain. We want to find a minimal projective resolution over $R$ of the moduli algebra $R / \overline{j(f)}$. Since $j(f)$ is $\mathfrak{m}_{S}$-primary the ideal $j(g) \subset k\left[\left|X_{1}, X_{2}\right|\right]$ is $\left(X_{1}, X_{2}\right)$-primary. Thus $g$ defines a formal plane curve with an isolated singularity. The moduli algebra $R / \overline{j(f)}$ is just $R /\left(\bar{X}_{3}^{n-1},{\overline{\partial g / \partial X_{1}}}_{1},{\overline{\partial g / \partial X_{2}}}_{2}\right.$. Similarly to what we did in the previous section, we will construct the desired resolution in a more general setting. Namely, let $\left(U, \mathfrak{m}_{U}\right)$ be a regular local ring of Krull dimension 2. Let $S:=U[|z|]$ be the formal power series ring in variable $z$ over $U$ and let $g \in \mathfrak{m}_{U}$. Suppose that $R:=S /\left(z^{n}+g\right)$ is an integrally closed domain. Finally, let $g_{1}, g_{2}$ be a system of parameters of $U$ such that $g_{1}$ (resp. $g_{2}$ ) is not a $U$-linear combination of $g$ and $g_{2}$ (resp. $g_{1}$ ). We want to find a 
minimal projective resolution of $R /\left(\bar{z}^{n-1}, \bar{g}_{1}, \bar{g}_{2}\right)$, where the overbars denote reduction modulo $\left(z^{n}+g\right)$.

We again begin with the case $g \in\left(g_{1}, g_{2}\right)$. Let $g=a_{1} g_{1}+a_{2} g_{2}, a_{1}, a_{2} \in$ $\mathfrak{m}_{U}$. Then $z^{n}+g=z \cdot z^{n-1}+a_{1} g_{1}+a_{2} g_{2}$, where $z, a_{1} a_{2} \in \mathfrak{m}_{S}$ and $z^{n-1}, g_{1}, g_{2}$, is a system of parameters. We can now apply Proposition 4.2 to obtain the desired resolution:

$$
\cdots \stackrel{\bar{\Phi}}{\rightarrow} R^{4} \stackrel{\bar{\Psi}}{\rightarrow} R^{4} \stackrel{\bar{\Phi}}{\rightarrow} R^{4} \stackrel{\bar{\Sigma}}{\rightarrow} R^{3} \stackrel{\bar{T}}{\rightarrow} R \rightarrow R /\left(\bar{z}^{n-1}, \bar{g}_{1}, \bar{g}\right) \rightarrow 0 .
$$

where

$$
\begin{gathered}
\Psi=\left(\begin{array}{cccc}
z & g_{2} & -g_{1} & 0 \\
a_{2} & -z^{n-1} & 0 & g_{1} \\
a_{1} & 0 & z^{n-1} & -g_{2} \\
0 & a_{1} & a_{2} & z
\end{array}\right), \quad \Phi=\left(\begin{array}{cccc}
z^{n-1} & g_{2} & g_{1} & 0 \\
a_{2} & -z & 0 & g_{1} \\
-a_{1} & 0 & z & g_{2} \\
0 & a_{1} & -a_{2} & z^{n-1}
\end{array}\right), \\
\Sigma=\left(\begin{array}{cccc}
z & g_{2} & -g_{1} & 0 \\
a_{2} & -z^{n-1} & 0 & g_{1} \\
a_{1} & 0 & z^{n-1} & -g_{2}
\end{array}\right), \quad \text { and } T=\left(z^{n-1} g_{1} g_{2}\right) .
\end{gathered}
$$

Now we assume that $g \notin\left(g_{1}, g_{2}\right)$. This implies that $g, g_{1}$ and $g_{2}$ are minimal generators of the ideal $\left(g, g_{1}, g_{2}\right) \subset U$. Then, as in the previous section, $\left(g_{1}, g_{2}\right):(g)=\left(b_{1}, b_{2}\right)$ and, if $\sigma:=\left(a_{i j}\right), i, j=1,2$, is such that

$$
{ }^{t} \sigma\left(\begin{array}{l}
g_{1} \\
g_{2}
\end{array}\right)=\left(\begin{array}{l}
b_{1} g \\
b_{2} g
\end{array}\right)
$$

then formulas (6.6)-(6.10) hold.

Using these formulas we immediately obtain the following complex:

$$
\ldots \stackrel{\bar{\alpha}_{3}}{\longrightarrow} R^{4} \stackrel{\bar{\alpha}_{4}}{\longrightarrow} R^{4} \stackrel{\bar{\alpha}_{3}}{\longrightarrow} R^{4} \stackrel{\bar{\alpha}_{2}}{\longrightarrow} R^{3} \stackrel{\bar{\alpha}_{1}}{\longrightarrow} R \rightarrow R /\left(\bar{g}_{1}, \bar{g}_{2}, \bar{z}^{n-1}\right) \rightarrow 0,
$$

where

$$
\begin{gathered}
\alpha_{4}=\left(\begin{array}{cccc}
a_{11} & a_{12} & z^{n-1} & 0 \\
a_{21} & a_{22} & 0 & z^{n-1} \\
-z & 0 & a_{22} & -a_{12} \\
0 & -z & -a_{21} & a_{11}
\end{array}\right), \\
\alpha_{3}=\left(\begin{array}{cccc}
a_{22} & -a_{12} & -z^{n-1} & 0 \\
-a_{21} & a_{11} & 0 & -z^{n-1} \\
z & 0 & a_{11} & a_{12} \\
0 & z & a_{21} & a_{22}
\end{array}\right), \\
\alpha_{2}=\left(\begin{array}{llll}
a_{11} & a_{12} & z^{n-1} & 0 \\
a_{21} & a_{22} & 0 & z^{n-1} \\
b_{1} z & b_{2} z & -g_{1} & -g_{2}
\end{array}\right),
\end{gathered}
$$

First we remark that $\left(\alpha_{3}, \alpha_{4}\right)$ is a matrix factorization of $z^{n}+g$. Therefore, this complex is exact in degree $>2$. 
To show that (7.1) is exact in degree 2 we have to prove the exactness of

$$
0 \rightarrow \operatorname{Im} \bar{\alpha}_{3} \stackrel{\beta_{2}}{\longrightarrow} R^{4} \stackrel{\beta_{1}}{\longrightarrow} \operatorname{Im} \bar{\alpha}_{2} \rightarrow 0,
$$

where $\beta_{2}$ is the natural inclusion and $\beta_{1}$ is the natural projection. To accomplish this we will employ the acyclicity Lemma 4.1. Let $I_{l}(-)$ denote the ideal generated by the $l \times l$ minors of the matrix "-". An easy calculation shows that $I_{3}\left(\bar{\alpha}_{2}\right)=0$. On the other hand, by $(6.10), 0 \neq \bar{g}=\operatorname{det}(\bar{\sigma}) \in I_{2}\left(\bar{\alpha}_{2}\right)$. Therefore, $\operatorname{rank} \operatorname{Im}\left(\bar{\alpha}_{2}\right)=2$. Another simple calculation shows that $\operatorname{det}\left(\alpha_{4}\right)=\left(z^{n}+g\right)^{2}$, yielding $2=\operatorname{rank} \operatorname{Coker} \bar{\alpha}_{4}=\operatorname{rank} \operatorname{Im} \bar{\alpha}_{3}=\operatorname{rank} \operatorname{Im} \beta_{2}$. Thus the modules in (7.2) have correct ranks. Next we check the reflexivity of $\operatorname{Im} \beta_{2}$ and $\operatorname{Ker} \beta_{1}$. Since $\operatorname{Ker} \beta_{1}=\operatorname{Ker} \bar{\alpha}_{2}$ which is the kernel of a map between reflexive modules, $\operatorname{Ker} \beta_{1}$ is reflexive. And since $\operatorname{Im} \beta_{2}=\operatorname{Im} \bar{\alpha}_{3}$ which comes from the matrix factorization $\left(\alpha_{4}, \alpha_{3}\right), \operatorname{Im} \beta_{2}$ is reflexive. Thus to apply the acyclicity lemma it remains to show that $\beta_{2}$ splits at the height 1 primes of $R$. The matrix factorization $\left(\alpha_{3}, \alpha_{4}\right)$ gives rise to the exact sequence

$$
0 \rightarrow \operatorname{Im} \bar{\alpha}_{3} \stackrel{\beta_{2}}{\longrightarrow} R^{4} \rightarrow \operatorname{Im} \bar{\alpha}_{4} \rightarrow 0,
$$

where $\operatorname{Im} \bar{\alpha}_{4} \cong \operatorname{Coker} \bar{\alpha}_{3}$ is reflexive. Localizing at height 1 primes we obtain the desired splitting. Thus complex (7.1) is exact in degree 2 .

Finally, to prove that (7.1) is exact in degree 1 we want to show that the complex

$$
0 \rightarrow \operatorname{Im} \bar{\alpha}_{2} \stackrel{\gamma_{2}}{\longrightarrow} R^{3} \stackrel{\gamma_{1}}{\longrightarrow} \operatorname{Im} \bar{\alpha}_{1} \rightarrow 0,
$$

where $\gamma_{2}$ is the natural inclusion and $\gamma_{1}$ is the natural projection, is exact. We already saw that $\operatorname{Im} \bar{\alpha}_{2} \cong \operatorname{Coker} \bar{\alpha}_{3}$ is a rank 2 reflexive. Since $\operatorname{Im} \alpha_{1}$ is a nonzero ideal of $R, \operatorname{rank} \operatorname{Im} \alpha_{1}=1$. And since $\bar{\alpha}_{1}$ is a map between reflexive modules, $\operatorname{Ker} \gamma_{1}=\operatorname{Ker} \alpha_{1}$ is reflexive. Thus to invoke the acyclicity lemma we have to show that for any height one prime $\mathfrak{p}$ of $R$ the map $\left(\gamma_{2}\right)_{\mathfrak{p}}$ is split or, equivalently, that $\left(\operatorname{Coker} \alpha_{2}\right)_{\mathfrak{p}}$ is $R_{\mathfrak{p}}$-free. Since $\operatorname{rank} \operatorname{Im} \bar{\alpha}_{2}=2$ we have to show that the ideal $I_{2}\left(\bar{\alpha}_{2}\right)$ of $2 \times 2$ minors of $\bar{\alpha}_{2}$ is $\mathfrak{m}_{R}$-primary or, equivalently, that height $\left(I_{2}\left(\bar{\alpha}_{2}\right)\right)=2$. So let $\mathfrak{q}$ be a prime containing $I_{2}\left(\bar{\alpha}_{2}\right)$. We alrelady saw that $\bar{g}=\operatorname{det}\left(\bar{a}_{i j}\right) \in I_{2}\left(\bar{\alpha}_{2}\right)$. Hence $\bar{z}^{n} \in \mathfrak{q}$.

Examining now $\bar{\alpha}_{2}$ we see that $\left(\bar{g}_{1}, \bar{g}_{2}\right)\left(\bar{a}_{11}, \bar{a}_{12}, \bar{a}_{21}, \bar{a}_{22}\right) \subset I_{2}\left(\bar{\alpha}_{2}\right) \subset \mathfrak{q}$. But (6.7) shows that $\left(\bar{g}_{1}, \bar{g}_{2}\right) \subset\left(\bar{a}_{11}, \bar{a}_{12}, \bar{a}_{21}, \bar{a}_{22}\right)$. It now follows that $\left(\bar{g}_{1}, \bar{g}_{2}\right) \subset \mathfrak{q}$ and $\mathfrak{q}$ contains an $\mathfrak{m}_{R}$-primary ideal $\left(\bar{g}_{1}, \bar{g}_{2}, \bar{z}^{n-1}\right)$. Thus $\operatorname{height}\left(I_{2}\left(\bar{\alpha}_{2}\right)\right)=2$ and $\gamma_{2}$ splits at height 1 primes. The acyclicity lemma shows that (7.1) is exact in degree 1. Since all the matrix elements in (7.1) belong to $\mathfrak{m}_{R}$ we obtain a minimal projective resolution over $R$ of $R /\left(\bar{g}_{1}, \bar{g}_{2}, \bar{z}^{n-1}\right)$.

Remark. It is possible to prove the exactness of (7.1) just by comparing the coefficients of the monomials in $z$ and without using the acyclicity lemma. The proof becomes a bit tedious but also shows that the assumptions that $U$ is noetherian and $R$ is integrally closed can be dropped. 
If we now set $S:=k\left[\left|X_{1}, X_{2}, X_{3}\right|\right]$, where $k$ is a field of characteristic 0 , $f:=X_{3}^{n}+g\left(X_{1}, X_{2}\right), n \geq 2$, where the jacobian ideal $j(f)=\left(X_{3}^{n-1}, \partial g / \partial X_{1}\right.$, $\left.\partial g / \partial X_{2}\right)$ is $\mathfrak{m}_{S}$-primary and $R:=S /(f)$, then, upon assuming, $f \notin j(f)$, we obtain a minimal presentation matrix of $D_{k}(R)^{*}$ (which is the second syzygy module of $R /\left(\bar{X}_{3}^{n-1}, \overline{\partial g / \partial X}_{1},{\overline{\partial g / \partial X_{2}}}_{2}\right)$ :

$$
R^{4}\left(\begin{array}{cccc}
\bar{a}_{22} & -\bar{a}_{12} & -\bar{X}_{3}^{n-1} & 0 \\
-\bar{a}_{21} & \bar{a}_{11} & 0 & -\bar{X}_{3}^{n-1} \\
\bar{X}_{3} & 0 & \bar{a}_{11} & \bar{a}_{12} \\
0 & \bar{X}_{3} & \bar{a}_{21} & \bar{a}_{22}
\end{array}\right) R^{4} \rightarrow D_{k}(R)^{*} \rightarrow 0,
$$

where the elements $a_{i j} \in k\left[\left|X_{1}, X_{2}\right|\right], i, j=1,2$, are defined (nonuniquely) by the equations

$$
\left(\begin{array}{ll}
a_{11} & a_{21} \\
a_{12} & a_{22}
\end{array}\right)\left(\begin{array}{l}
\partial g / \partial X_{1} \\
\partial g / \partial X_{2}
\end{array}\right)=\left(\begin{array}{l}
b_{1} g \\
b_{2} g
\end{array}\right)
$$

where $b_{1}$ and $b_{2}$ are minimal generators of the ideal

$$
\left(\partial g / \partial X_{1}, \partial g / \partial X_{2}\right):(g) \subset k\left[\left|X_{1}, X_{2}\right|\right] .
$$

\section{A PROOF OF THE MAIN THEOREM}

We now return to Theorem 3.2. Suppose first that $f \in j(f)$. Then by Proposition 3.1 the factor algebra $S /(f)$ is positively graded. But $S /(f)$ is also integrally closed and Gorenstein. By Corollary 2.3 the Auslander module $A$ is isomorphic to the module $D_{k}(R)^{* *}$ of Zariski differentials.

Conversely, suppose that $A$ is isomorphic to $D_{k}(R)^{* *}$. We will show that the assumption $f \notin j(f)$ leads to a contradiction. For this purpose we need the following result of Scheja and Wiebe:

Proposition 8.1 [SchW1, 3.1]. Let $R$ be a two-dimensional integrally closed analytic $k$-algebra, where $k$ is an algebraically closed field of characteristic 0 . If there exists a nonnilpotent derivation of $R$ into itself then $R$ is positively graded.

Recall that a $k$-derivation $\delta: R \rightarrow R$ sending the maximal ideal $\mathfrak{m}_{R}$ into itself is called nilpotent if for any $\nu \in \mathbf{N}$ the induced derivation of $R / \mathfrak{m}_{R}^{\nu+1}$ into itself is nilpotent. Our aim now is to construct a nonnilpotent derivation of $R$ into itself.

By Corollary 5.2 the module $D_{k}(R)^{* *}$ is isomorphic to the module $D_{k}(R)^{* *}$. Thus the minimal presentation matrices of $A(4.1)$ and $D_{k}(R)^{*}(7.3)$ are equivalent:

$$
\overline{\mathbf{\Phi}}=\left(\begin{array}{cccc}
\bar{f}_{3} & \bar{f}_{2} & \bar{f}_{1} & 0 \\
\bar{X}_{2} & -\bar{X}_{3} & 0 & \bar{f}_{1} \\
-\bar{X}_{1} & 0 & \bar{X}_{3} & \bar{f}_{2} \\
0 & \bar{X}_{1} & -\bar{X}_{2} & \bar{F}_{3}
\end{array}\right) \sim \bar{\alpha}_{3}=\left(\begin{array}{cccc}
\bar{a}_{22} & -\bar{a}_{12} & -\bar{X}_{3}^{n-1} & 0 \\
-\bar{a}_{21} & \bar{a}_{11} & 0 & -\bar{X}_{3}^{n-1} \\
\bar{X}_{3} & 0 & \bar{a}_{11} & \bar{a}_{12} \\
0 & \bar{X}_{3} & \bar{a}_{21} & \bar{a}_{22}
\end{array}\right) .
$$


Here $f=X_{1} f_{1}+X_{2} f_{2}+X_{3} f_{3}$ and the elements $a_{i j} \in k\left[\left|X_{1}, X_{2}\right|\right], i, j=1,2$, are defined (nonuniquely) by the equations

$$
\left(\begin{array}{ll}
a_{11} & a_{21} \\
a_{12} & a_{22}
\end{array}\right)\left(\begin{array}{l}
\partial g / \partial X_{1} \\
\partial g / \partial X_{2}
\end{array}\right)=\left(\begin{array}{l}
b_{1} g \\
b_{2} g
\end{array}\right)
$$

where $b_{1}$ and $b_{2}$ are minimal generators of the ideal

$$
\left(\partial g / \partial X_{1}, \partial g / \partial X_{2}\right):(g) \subset\left(X_{1}, X_{2}\right) \subset k\left[\left|X_{1}, X_{2}\right|\right] .
$$

(The equivalence relation is given by multiplication by invertible matrices on both sides.)

Since equivalent matrices have the same Fitting ideals, the ideals generated by the entries of $\bar{\Phi}$ and $\bar{\alpha}_{3}$ are equal. Hence the images $\bar{a}_{i j}$ of the elements of $a_{i j}$ generate the maximal ideal of $k\left[\left|X_{1}, X_{2}\right|\right] /(g)$. Since the jacobian ideal $j(g)$ is primary to the maximal ideal $\left(X_{1}, X_{2}\right)$ of $k\left[\left|X_{1}, X_{2}\right|\right]$ we have $g \in\left(X_{1}, X_{2}\right)^{2}$. Therefore the elements $a_{i j}, i, j=1,2$, generate $\left(X_{1}, X_{2}\right)$. Thus we have a matrix

$$
\sigma=\left(\begin{array}{ll}
a_{11} & a_{12} \\
a_{21} & a_{22}
\end{array}\right)
$$

such that $\operatorname{det} \sigma=g($ see $(6.10))$ and $\left(a_{11}, a_{12}, a_{21}, a_{22}\right)=\left(X_{1}, X_{2}\right)$.

We claim that the multiplicity (i.e., the degree of the lowest order terms) of $g$ is at least 3. Otherwise using the Weierstrass preparation theorem and the Tschirnhausen transformation (i.e., extracting a perfect square) we could write $g=y_{1}^{2}+h\left(y_{2}\right)$ for some new variables $y_{1}$ and $y_{2}$. If $t \geq 1$ is the lowest power of $y_{2}$ in $h$ then $h\left(y_{2}\right)=y_{2}^{t} \cdot u$, where $u$ is a unit. But then $g \in j(g)$ and, therefore, $f \in j(f)$, contrary to the assumption $f \notin j(f)$.

Since the elements $a_{i j}$ generate $\left(X_{1}, X_{2}\right)$ we can find two of these elements with the same property. Without loss of generality we can assume that $a_{11}$ is one of them. We now have the following three cases:

Case 1. The elements $a_{11}$ and $a_{12}$ generate $\left(X_{1}, X_{2}\right)$. Then we can find $\lambda_{1}, \lambda_{2} \in k$ such that $\lambda_{1} a_{11}+\lambda_{2} a_{12} \equiv X_{1} \bmod \left(X_{1}, X_{2}\right)^{2}$. From (8.1) we obtain derivations

$$
\delta_{i}:=\bar{a}_{1 i} \frac{\partial}{\partial X_{1}}+\bar{a}_{2 i} \frac{\partial}{\partial X_{2}}, \quad i=1,2,
$$

of $k\left[\left|X_{1}, X_{2}\right|\right] /(g)$ into itself. Let $\delta:=\lambda_{1} \delta_{1}+\lambda_{2} \delta_{2}$. Then

$$
\delta\left(\bar{X}_{1}\right) \equiv \bar{X}_{1} \bmod \left(\bar{X}_{1}, \bar{X}_{2}\right)^{2}
$$

and $\delta$ is not nilpotent. Lifting $\delta$ in the obvious way to a nonnilpotent derivation of $R$ we deduce from Proposition 8.1 that $R$ is graded, contrary to the assumption $f \notin j(f)$.

Case 2. The elements $a_{11}$ and $a_{21}$ generate $\left(X_{1}, X_{2}\right)$. Consider the derivation

$$
\delta:=\bar{a}_{11} \frac{\partial}{\partial X_{1}}+\bar{a}_{21} \frac{\partial}{\partial X_{2}}
$$


of $k\left[\left|X_{1}, X_{2}\right|\right] /(g)$. Then the reduction of $\delta$ modulo $\left(\bar{X}_{2}, \bar{X}_{2}\right)^{2}$ is an isomorphism. Lifting it to $R$ in the obvious way we again obtain a contradiction.

Case 3. The elements $a_{11}$ and $a_{22}$ generate $\left(X_{1}, X_{2}\right)$ and none of the previous two conditions holds. Let $L\left(a_{i j}\right)$ denote the linear part of $a_{i j}, i, j=1,2$. Then $L\left(a_{21}\right)=\lambda_{1} L\left(a_{11}\right), L\left(a_{12}\right)=\lambda_{2} L\left(a_{11}\right)$, and $\left(L\left(a_{11}\right), L\left(a_{22}\right)\right)=\left(X_{1}, X_{2}\right)$ for some $\lambda_{1}, \lambda_{2} \in k$. Since $\operatorname{det} \sigma=g$ and $\operatorname{mult}(g) \geq 3$ we must have

$$
\left|\begin{array}{cc}
L\left(a_{11}\right) & \lambda_{2} L\left(a_{11}\right) \\
\lambda_{1} L\left(a_{11}\right) & L\left(a_{22}\right)
\end{array}\right|=0
$$

or $L\left(a_{11}\right) L\left(a_{22}\right)-\lambda_{1} \lambda_{2} L\left(a_{11}\right)^{2}=0$. Since $L\left(a_{11}\right)$ is different from zero, $L\left(a_{22}\right)$ $=\lambda_{1} \lambda_{2} L\left(a_{11}\right)$. But then $L\left(a_{11}\right)$ and $L\left(a_{22}\right)$ could not generate the maximal ideal $\left(X_{1}, X_{2}\right)$, contrary to our assumption. This finishes the proof of Theorem 3.2 .

An immediate consequence of the just proved result is that $D_{k}(R)^{* *}$ and $A$ are not always isomorphic. Indeed take $g \in k\left[\left|X_{1}, X_{2}\right|\right]$ to be $X_{1}^{5}+X_{2}^{5}+X_{1}^{3} X_{2}^{3}$ and set $f=X_{3}^{2}+X_{1}^{5}+X_{2}^{5}+X_{1}^{3} X_{2}^{3}$. Then $g \notin j(g)$ and, therefore, $f \notin j(f)$. Hence $D_{k}(R)^{* *}$ is not isomorphic to $A$.

Notice that in the proof of Theorem 3.2 we also proved

Corollary 8.2. Let $S=k\left[\left|X_{1}, X_{2}\right|\right]$ be the formal power series ring over an algebraically closed field $k$ of characteristic 0 . Let $g \in \mathfrak{m}_{S}$ be a formal power series whose jacobian ideal $j(g)$ is $\mathfrak{m}_{S}$-primary and suppose that $g \notin j(g)$. Then the ideal of $R:=S /(g)$ generated by the entries of the relation matrix of an arbitrary syzygy module of the $R$-module $R / \overline{j(g)}$ is strictly contained in the maximal ideal of $R$.

Remark. Let mult $(g)=n$ and let $I(g)$ denote the sum of the monomials of $g$ of order $n$. If we assume that $I(g)$ is not an $n$th power then the condition that $k$ be algebraically closed can be dropped from Corollary 8.2. The proof is based on the investigation of the Newton polygon of $g$, which allows us to bypass the result of Scheja and Wiebe on nonnilpotent derivations.

\section{REFERENCES}

[Aus1] M. Auslander, Rational singularities and almost split sequences, Trans. Amer. Math. Soc. 293 (1986), 511-531.

[Aus2] _ Coherent functors, Proc. Conf. Categorical Algebra, Springer-Verlag, 1966, pp. 189231.

[Aus3] _ Almost split sequences and algebraic geometry, Representation of Algebras, Proc. Durham Symposium, Cambridge Univ. Press, 1986, pp. 165-179.

[AB] M. Auslander and D. Buchsbaum, On ramification theory in noetherian rings, Amer. J. Math. 81 (1959), 749-765.

[AR] M. Auslander and I. Reiten, Almost split sequences in dimension two, Adv. in Math. 66 (1987), 88-118.

[B-AC] N. Bourbaki, Algèbre commutative, Chapitre 7, Hermann, Paris, 1965.

$\left[\mathrm{B}-\mathrm{AC}^{\prime}\right]$ Algèbre commutative, Chapitres 8 et 9, Masson, Paris, 1983. 
[B-E] D. Buchsbaum and D. Eisenbud, Algebra structure for finite free resolutions and some structure theorems for ideals of codimension 3, Amer. J. Math. 99 (1977), 447-485.

[D] D. Eisenbud, Homological algebra on a complete intersection, Trans. Amer. Math. Soc. 260 (1980), 35-64.

[Ho] M. Hochster, The Zariski-Lipman conjecture in the graded case, J. Algebra 47 (1977), $411-$ 424.

[Nag] M. Nagata, Local rings, Krieger, 1975.

[Nar] I. Naruki, Some remarks on isolated singularity and their application to algebraic manifolds, Publ. Res. Inst. Math. Sci. 13 (1977), 17-46.

[PS] C. Peskine and L. Szpiro, Liaison des variétés algébriques, Invent. Math. 26 (1974), 271302.

[P1] E. Platte, Operation von endlichen Gruppen auf Differentialen, Dissertation, Universität Osnabrück, Juni 1977.

[P2] - Ein elementarer Bewis das Zariski-Lipman-Problems für graduirte analytische Algebren, Arch. Math. 31 (1978), 143-145.

[Sai] K. Saito, Quasihomogene isolierte Singularitäten von Hyperflächen, Invent. Math. 14 (1971), 123-142.

[Sch] G. Scheja, Differential modules of analytic rings, Lectures given at University of Genova, 1968.

[SchSt] G. Scheja and U. Storch, Differentielle Eigenschafften der Lokalisierungen analytischer Algebren, Math. Ann. 197 (1972), 137-170.

[SchW1] G. Scheja and H. Wiebe, Zur Chevalley-Zerlegung von Derivationen, Manuscripta Math. 33 (1980), 159-176.

[SchW2] __ Über Derivationen von lokalen analytische Algebren, Sympos. Math. 11 (1973), 161192.

[Se] J.-P. Serre, Sur les modules projectifs, Sém. Dubreil, 1960/1961.

[Tate] J. Tate, Homology of Noetherian rings and local rings, Illinois J. Math. 1 (1957), 14-27.

[Te] B. Teissier, The hunting of invariants in the geometry of discriminants, Proc. Nordic Summer School, Oslo, 1976, pp. 565-677.

[Wahl] J. Wahl, The jacobian algebra of a graded Gorenstein singularity, Duke Math. J. 55 (1987), 843-871.

Department of Mathematics, Northeastern University, Boston, Massachusetts 02115 